\title{
Mechanism of Cyclopolymerization of Vinyl Acrylate, Methacrylate, and $\alpha$-Chloroacrylate
}

\author{
Wakichi FUKUDA, Yutaka YAMANO, Masaaki TsURIYA, \\ and Hiroshi KAKIUCHI \\ Department of Applied Chemistry, Faculty of Engineering, \\ Yokohama National University, \\ 156 Tokiwadai, Hodogaya-ku, Yokohama 240, Japan.
}

(Received June 24, 1981)

\begin{abstract}
Polymers of vinyl acrylate, methacrylate, and $\alpha$-chloroacrylate were prepared by radical polymerization at $60^{\circ} \mathrm{C}$ at various monomer concentrations in benzene. The residual double bond in the polymers was determined to be mainly a vinyl group by infrared spectroscopy and ICl titrimetry. The decrease in the residual double bond with decreasing monomer concentration can be explained in terms of an intramolecular chain monocyclization-bicyclization mechanism. The proposed monocyclization was carried out between the polymer radical and vinyl group of a penultimate monomer unit. Bicyclization was carried out between a monocyclized radical and vinyl group of the polymer end. The ratios of monocyclization rate constant over intermolecular propagation rate constant were evaluated to be 20 for vinyl acrylate, 0.22 for vinyl methacrylate and $0.60 \mathrm{~mol} \mathrm{dm}^{-3}$ for vinyl $\alpha$-chloroacrylate, respectively. The ratios of bicyclization rate constant over intermolecular propagation rate constant were evaluated to be $0.1 \mathrm{~mol} \mathrm{dm}^{-3}$ for all the monomers.
\end{abstract}

KEY WORDS Cyclopolymerization / Monocyclization / Bicyclization / Vinyl Acrylate / Vinyl Methacrylate / Vinyl $\alpha$-Chloroacrylate / Cyclocopolymerization /

In the cyclopolymerization of unsymmetrical nonconjugated 1,5-dienes, vinyl $\beta$-substituted acrylates were shown to give polymers containing $\gamma$ lactone units along the polymer chain. ${ }^{1-3}$ On the other hand, we showed that both vinyl acrylate (VA) and vinyl methacrylate (VMA) were polymerized by free radical initiator to give polymers containing $\delta$-lactone or larger cyclic units. ${ }^{4}$ Guaita et al. have proposed that a poly(VMA) obtained by radical polymerization contained $\gamma$-lactone unit as a result of kinetic consideration, though they did not show any characteristic support for this structure. ${ }^{5}$

Butler has reconsidered certain aspects of cyclopolymerization mechanism of 1,6-dienes and showed that numerous examples of such polymerizations lead to large fractions of five-membered rings although six-membered rings were predicted on the basis of radical stabilities. ${ }^{6}$ Johns et al. ${ }^{7}$ as well as Tsukino and Kunitake ${ }^{8}$ showed ${ }^{13} \mathrm{C}$ NMR spectroscopy to be very useful in determining the ring size of cyclopolymers. The ring size in the cyclopolymerization of VA and VMA remains to be clarified in terms of experimental results.

In a previous paper, we proposed a mechanism for the cyclopolymerization of VA and $\mathrm{VMA},{ }^{4}$ in which intermolecular chain propagation was carried out mainly between growing polymer radicals and acrylic or methacrylic groups of monomers, and the intramolecular monocyclization between growing polymer radicals and vinyl groups of a penultimate monomer unit followed the intramolecular bicyclization between monocyclized radicals and the vinyl groups of terminal monomer unit.

In order to obtain additional support for the proposed mechanism, an attempt was made to evaluate the kinetics of cyclopolymerization and to determine cyclization constants. In addition, polymerization and copolymerization of vinyl $\alpha$-chloroacrylate (VCA) were carried out. The effect of the $\alpha$-substituent of acrylates on the extent of 


\section{W. FUKUDA et al.}

cyclization is discussed.

\section{EXPERIMENTAL}

\section{Monomers}

VA and VMA were prepared as described in a previous paper. ${ }^{4}$ VCA was prepared by a vinyl interchange reaction between vinyl acetate and $\alpha$ chloroacrylic acid prepared according to Marvel's method. ${ }^{9}$ Thus, $21 \mathrm{~g}$ of $\alpha$-chloroacrylic acid, $80 \mathrm{~g}$ of vinyl acetate, $2.5 \mathrm{~g}$ of hydroquinone and $0.8 \mathrm{~g}$ of mercury(II) acetate were placed in a three necked flask equipped with stirrer, thermometer and reflux condenser. To this mixture was added dropwise $0.4 \mathrm{~cm}^{3}$ of $\mathrm{BF}_{3} \cdot \mathrm{O}\left(\mathrm{C}_{2} \mathrm{H}_{5}\right)_{2}$ with vigorous stirring. After $6 \mathrm{~h}$ of reaction at $30^{\circ} \mathrm{C}$, the mixture was neutralized with aqueous $\mathrm{Na}_{2} \mathrm{CO}_{3}$, and washed with aqueous $\mathrm{NaHCO}_{3}$ and distilled water repeatedly. The organic layer was dried over anhydrous sodium sulfate. Redistillation gave the fraction boiling at $39^{\circ} \mathrm{C} / 19 \mathrm{mmHg}$ (yield $30 \%$ ); $n_{\mathrm{D}}^{25} 1.4610 ; d_{20}^{20} 1.1540$; IR 1740 (ester $\mathrm{C}=\mathrm{O}$ ), 1640 (vinyl $\mathrm{C}=\mathrm{C}$ ), and 1615 $\mathrm{cm}^{-1}(\alpha$-chloroacryl C $=\mathrm{C})$; NMR (neat) $4.61(\mathrm{~d}, 1$, $\left.J=6.1 \mathrm{~Hz}, c i s-\mathrm{CH}_{2}=\mathrm{CH}\right), 4.93(\mathrm{~d}, 1, J=13.8 \mathrm{~Hz}$, trans $\left.-\mathrm{CH}_{2}=\mathrm{CH}\right), 6.01\left(\mathrm{~s}, 1\right.$, cis $\left.-\mathrm{CH}_{2}=\mathrm{CCl}\right), 6.49(\mathrm{~s}$, 1 , trans $\left.-\mathrm{CH}_{2}=\mathrm{CCl}\right)$, and $7.13 \mathrm{ppm}(\mathrm{q}, 1,=\mathrm{CH}-)$. Anal. Calcd for $\mathrm{C}_{5} \mathrm{H}_{5} \mathrm{O}_{2} \mathrm{Cl}: \mathrm{C}, 45.32 \% ; \mathrm{H}, 3.77 \%$; $\mathrm{Cl}, 26.79 \%$. Found: C, $45.19 \% ; \mathrm{H}, 4.08 \% ; \mathrm{Cl}$, $27.05 \%$.

Monomers were redistilled prior to use and their purity was determined to be $99 \%$ or higher by GLPC where the liquid phase was diethylene glycol succinate.

\section{Other Reagents}

Other monomers were obtained commercially and purified in the usual manner. Solvents and the initiator were purified as usual.

\section{Radical Polymerization}

This was conducted by the same procudure described in the previous paper. ${ }^{4}$ Poly(VCA) was obtained as white powder.

Anal. Calcd for $\mathrm{C}_{2} \mathrm{H}_{5} \mathrm{O}_{2} \mathrm{Cl}: \mathrm{C}, 45.32 \% ; \mathrm{H}, 3.77 \%$; $\mathrm{Cl}, 26.79 \%$. Found: C, $45.79 \% ; \mathrm{H}, 4.43 \% ; \mathrm{Cl}$, $26.92 \%$.

\section{Anionic Polymrization of $V C A$}

A VCA solution $\left(1.4 \mathrm{~mol} \mathrm{dm}^{-3}\right)$ in toluene was placed in a glass tube equipped with a neoprene rubber stopper. The operation was carried out in a dry-box filled with nitrogen. $4.4 \mathrm{~mol} \%$ of $n$-butyl Li (provided by Sankyo Kasei Co. Ltd. as $15.2 \mathrm{wt} \%$ solution in $n$-hexane) per VCA was added through a hypodermic syringe at $-78^{\circ} \mathrm{C}$. The polymerization was continued for 100 minutes and stopped by adding small amount of methanol. The polymer was separated by reprecipitation in methanol and dried in vacuo at room temperature (yield $7.1 \%$ ). $\eta_{\mathrm{sp}} / c$, $0.11 \times 10^{2} \mathrm{~cm}^{3} \mathrm{~g}^{-1}$ in toluene at $30^{\circ} \mathrm{C}$. The low yield was analogous to the anionic polymerization of methyl $\alpha$-chloroacrylate. ${ }^{10}$

\section{Analyses}

The residual double bond (RDB) in the polymer was determined by the $\mathrm{ICl}$ titrimetric method in which chloroform or dioxane is used as the solvent. The reaction time was one hour. IR spectra were recorded on a Hitachi EPI-2 spectrometer and ${ }^{1} \mathrm{H}$ NMR spectra were recorded on a JEOL-JNM C$60 \mathrm{H}$ spectrometer with tetramethylsilane as the internal reference.

\section{RESULTS AND DISCUSSION}

\section{Characterization of Poly $(V C A)$}

Figure 1 shows the IR spectra of VCA monomer and poly(VCA). The absorption band at $1651 \mathrm{~cm}^{-1}$ attributed to acrylic double bond in monomer can hardly be observed in either IR spectra of anionic

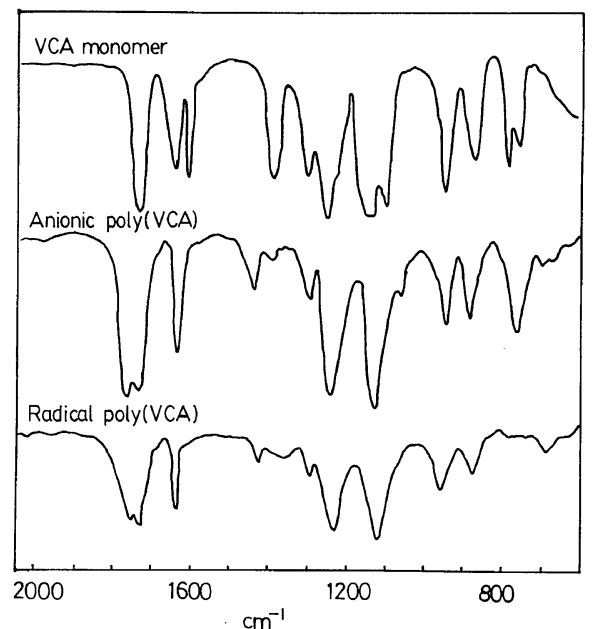

Figure 1. Infrared spectra of VCA monomer and polymers: monomer, liquid film; anionic poly(VCA), film; radical poly(VCA), $\mathrm{KBr}$ disk. 
and radical poly(VCA). The absorption band at $1640 \mathrm{~cm}^{-1}$, attributed to vinyl $\mathrm{C}=\mathrm{C}$ stretching vibration, can be seen in the IR spectra of both polymers. Thus, the polymer chains are considered to consist mainly of acrylic sequences.

The absorption band attributed to ester carbonyl splits into two bands of unsymmetrical intensity, a minor band at $1733 \mathrm{~cm}^{-1}$ and a major band at 1760 $\mathrm{cm}^{-1}$ for anionic poly(VCA). For radical poly(VCA), there is a major band at $1735 \mathrm{~cm}^{-1}$ and a minor band at $1761 \mathrm{~cm}^{-1}$. The explanation for the splitting of the carbonyl absorption band of $\alpha$ halogenated esters according to Bellamy and Williams ${ }^{11}$ is the presence of rotational isomers. The difference in the intensities of the two resolved carbonyl bands is attributed to the tacticities pointed out by Wesslen and Lenz in the case of poly(ethyl $\alpha$ chloroacrylate). ${ }^{12}$ According to their analysis, Figure 1 suggests that the anionic poly(VCA) is mainly isotactic and radical poly(VCA) is moderately syndiotactic. Therefore, the carbonyl absorption band at $1760 \mathrm{~cm}^{-1}$ for the radical polymer is no proof of the structure of $\gamma$-lactone unit.

${ }^{1} \mathrm{H}$ NMR spectrum of the anionic poly(VCA) in $\mathrm{CDCl}_{3}$ does not show a peak for acrylic protons but shows chemical shifts at $6.93(\mathrm{q}, 1), 4.93(\mathrm{~d}, 1)$, and

Table I. Determination of vinyl groups in monomers by $\mathrm{ICl}$ titration $^{\mathrm{a}}$

\begin{tabular}{cc}
\hline & Vinyl group fraction \\
\cline { 2 - 2 } Monomer & mol per monomer \\
\hline VA & 0.969 \\
VMA & 1.036 \\
VCA & 0.970 \\
Vinyl acetate & 0.989 \\
\hline
\end{tabular}

a Reaction was carried out for $1 \mathrm{~h}$ in chloroform.

Table II. Radical polymerization of VA, VMA, and VCA in benzene at $60^{\circ} \mathrm{C}^{\mathrm{a}}$

\begin{tabular}{|c|c|c|c|c|c|}
\hline \multirow{2}{*}{ Monomer } & \multirow{2}{*}{$\frac{[\mathrm{M}]_{0}}{\mathrm{~mol} \mathrm{dm}}$} & \multirow{2}{*}{$\frac{\text { Time }}{\min }$} & \multirow{2}{*}{$\frac{\text { Yield }}{w t^{\circ} \%}$} & \multirow{2}{*}{$\frac{\eta_{\mathrm{sp}} / c^{\mathrm{b}}}{100 \mathrm{~cm}^{3} \mathrm{~g}^{-1}}$} & $\mathrm{RDB}^{\mathrm{c}}$ \\
\hline & & & & & mol per monomer unit \\
\hline \multirow[t]{6}{*}{ VA } & 0.20 & 30 & 26.6 & 0.04 & $0.36(0.32)$ \\
\hline & 0.40 & 20 & 12.6 & 0.07 & $0.40(0.41)$ \\
\hline & 0.60 & 15 & 5.7 & 0.11 & $0.39(0.43)$ \\
\hline & 0.80 & 15 & 7.2 & 0.09 & $0.44(0.46)$ \\
\hline & 1.00 & 15 & 4.8 & 0.06 & $0.47(0.47)$ \\
\hline & 1.20 & 10 & 4.1 & - & $0.48(0.45)$ \\
\hline \multirow[t]{8}{*}{ VMA } & 0.25 & 60 & 11.1 & 0.07 & $0.57(0.55)$ \\
\hline & 0.50 & 60 & 15.0 & 0.13 & $0.71(0.70)$ \\
\hline & 0.77 & 60 & 21.6 & 0.21 & $0.79(0.78)$ \\
\hline & 1.00 & 60 & 16.4 & 0.27 & $0.84(0.78)$ \\
\hline & 1.25 & 60 & 21.3 & 0.39 & $0.87(0.89)$ \\
\hline & 1.50 & 30 & 9.0 & 0.47 & $0.89(0.93)$ \\
\hline & 1.75 & 30 & 9.1 & 0.67 & $0.92(0.99)$ \\
\hline & 2.00 & 20 & 5.3 & 0.72 & $0.91(0.84)$ \\
\hline \multirow[t]{8}{*}{ VCA } & 0.19 & 30 & 8.5 & 0.03 & 0.48 \\
\hline & 0.40 & 30 & 11.4 & 0.07 & 0.53 \\
\hline & 0.50 & 30 & 12.4 & 0.05 & 0.57 \\
\hline & 0.69 & 30 & 12.3 & 0.09 & 0.65 \\
\hline & 0.78 & 30 & 13.4 & 0.13 & 0.70 \\
\hline & 1.02 & 30 & 12.6 & 0.16 & 0.72 \\
\hline & 1.32 & 30 & 12.7 & 0.26 & 0.73 \\
\hline & 1.59 & 30 & 12.8 & 0.40 & 0.78 \\
\hline
\end{tabular}

${ }^{\mathrm{a}}$ [AIBN], $0.3 \times 10^{-2} \mathrm{~mol} \mathrm{dm}{ }^{-3}$ for VA; $1.0 \times 10^{-2} \mathrm{~mol} \mathrm{dm}^{-3}$ for VMA and VCA.

${ }^{\mathrm{b}}$ Determined in benzene at $25^{\circ} \mathrm{C} ; c, 0.25-0.50 \mathrm{~g} \mathrm{dl}^{-1}$.

c Determined by ICl titration and IR spectroscopy as shown in parenthesis. 
$4.64(\mathrm{~d}, 1) \mathrm{ppm}$ for vinyl protons. On the other hand, the ${ }^{1} \mathrm{H}$ NMR spectrum of radical poly(VCA) shows broads peaks.

\section{$R D B$ in Radical Polymers}

Table I shows the results for the determination of the vinyl double bond in monomers. The vinyl group can be determined by the ICl titrimetric method within an experimental error of $\pm 3 \%$. Table II shows the results of the polymerization of VA, VMA, and VCA as well as RDB in the polymers. On adding $\mathrm{ICl}$, the polymers show no absorption band between 1640 and $1645 \mathrm{~cm}^{-1}$ in their IR spectra.

RDB in poly(VA) and poly(VMA) was also determined by IR spectroscopy as described in the previous paper. ${ }^{4}$ The RDB value of each polymer determined by $\mathrm{ICl}$ titrimetry is in agreement with that determined by IR spectroscopy within experimental error. Consequently, it may be said that all of the radical polymers contain vinyl double bonds to a substantial extent. Guaita et al. determined that the methacrylic double bond in the radical poly(VMA) was in the range of $0.05-0.22 \mathrm{~mol}$ per monomer unit. $^{5}$

\section{Cyclization Mechanism at Growing Polymer End}

When there is an intermolecular chain propagation between the growing polymer radical and acrylic or $\alpha$-substituted acrylic group in monomers, an intramolecular cyclization reaction may take place between the growing polymer radical and the pendent vinyl group of an end unit. Such a cyclization reaction should lead to the formation of $\gamma$ lactone structural units not observable in IR spectra of the radical polymers. Even though there is no proof for this structure, the equation derived by Smets and Roovers for the cyclopolymerization of vinyl cinnamate ${ }^{1}$ may be applied to the present study.

Figure 2 shows the relationships between 1/ $\left(1-f_{\mathrm{u}}\right)$ and initial monomer concentration $[\mathrm{M}]_{0}$ in the polymerization of VA, VMA, and VCA. The plots for VMA satisfy equation, ${ }^{1}$

$$
\frac{1}{1-f_{\mathrm{u}}}=1+\frac{[\mathrm{M}]_{0}}{r_{\mathrm{c}}}
$$

where $f_{\mathrm{u}}$ is a fraction of RDB per monomer unit, $r_{\mathrm{c}}$ is $k_{\mathrm{c}} / k_{\mathrm{x}}$ in which $k_{\mathrm{c}}$ and $k_{\mathrm{x}}$ are the rate constants for intramolecular cyclization and intermolecular propagation, respectively. The cyclization constant $r_{\mathrm{c}}$ was calculated to be $0.19 \mathrm{~mol} \mathrm{dm}^{-3}$ for VMA. Neither of the plots for VA and VCA follow eq 1 . This suggest that a cyclization mechanism of a growing polymer end is improbable.

\section{Bicyclization Mechanism}

When the intramolecular cyclization consists of

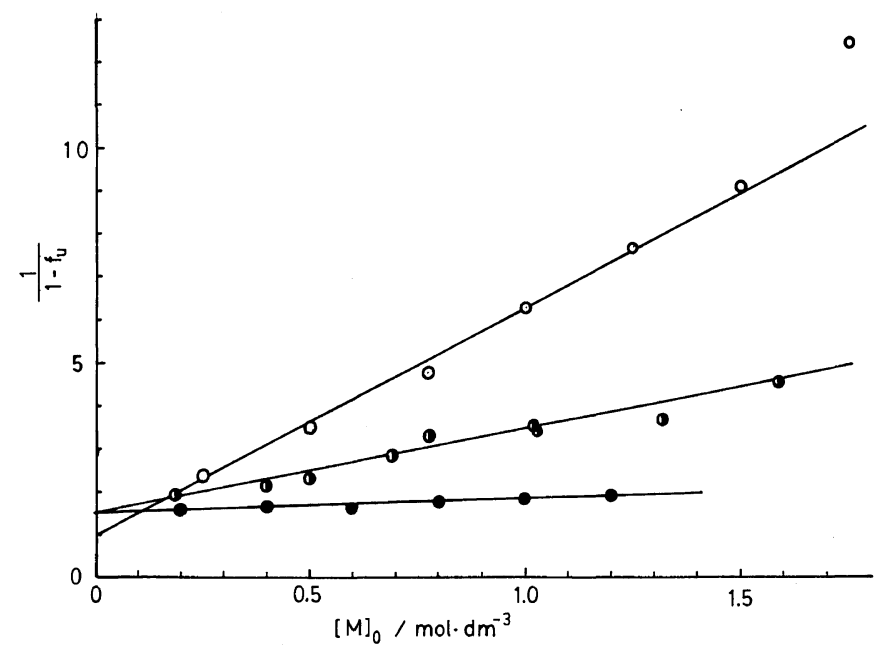

Figure 2. Effect of initial monomer concentration $[\mathrm{M}]_{0}$ on the reciprocal of cyclized fraction $\left(1-f_{\mathrm{u}}\right)$ in the polymer obtained at $60^{\circ} \mathrm{C}$, $[\mathrm{AIBN}]_{0}, 3 \times 10^{-3} \mathrm{~mol} \mathrm{dm}{ }^{-3}$ for VA, $1.0 \times 10^{-2} \mathrm{~mol} \mathrm{dm}^{-3}$ for VMA and VCA in benzene: $\bigcirc$, VA; $\bigcirc$, VMA, $\bigcirc$, VCA. 
two steps, the first step is the monocyclization of a growing polymer radical with a vinyl group of a penultimate monomer unit and the second step is the bicyclization of a monocyclized radical with a

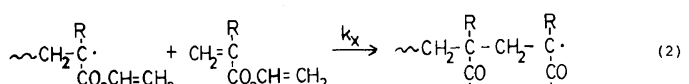

$$
\begin{aligned}
& \begin{array}{ll}
\mathrm{M} & \stackrel{\mathrm{O}}{\mathrm{C}} \mathrm{H}=\mathrm{CH}_{2} \stackrel{\dot{\mathrm{C}}}{\mathrm{C}} \mathrm{H}=\mathrm{CH}_{2}
\end{array}
\end{aligned}
$$

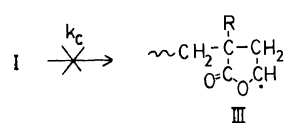

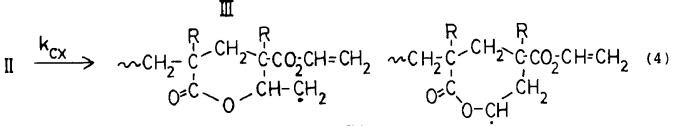

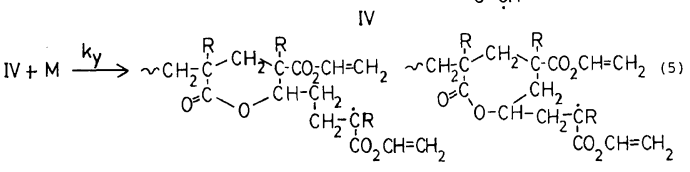

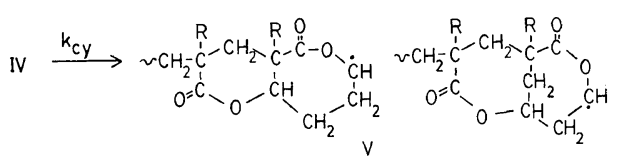

$$
\begin{aligned}
& \text { VA } R: H \\
& \text { VMA R: } \mathrm{CH}_{3} \\
& \text { VCA } \mathrm{R}: \mathrm{Cl}
\end{aligned}
$$

Scheme I.

vinyl group of a polymer terminal. Scheme I shows the proposed reaction. The saturated monomer units along the polymer chain will be formed in the reactions of eq 4 and 6 . Two more bicyclic structures are considered in addition to the bicyclic units shown in eq 6 . These cyclic structures remain to be elucidated with respect to polymer characterization, e.g., ${ }^{13} \mathrm{C}$ NMR spectroscopy. ${ }^{7.8}$ The bicyclization mechanism is similar to that for the cyclopolymerization of divinyl ethers, as symmetrical and unsymmetrical 1,4-dienes. ${ }^{8,13-15}$

The kinetics derived by Guaita et al. ${ }^{13}$ are applied to the present study as follows. When saturated and unsaturated monomer units in the polymer chain are designated as $S$ and $U$, and the concentrations of growing polymer radicals II and IV as [X·] and $[\mathrm{Y} \cdot]$, respectively, their formation rates may be expressed as,

$$
\frac{\mathrm{d} S}{\mathrm{~d} t}=k_{\mathrm{cx}}[\mathrm{X} \cdot]+k_{\mathrm{cy}}[\mathrm{Y} \cdot]
$$

$$
\frac{\mathrm{d} U}{\mathrm{~d} t}=k_{\mathrm{x}}[\mathrm{X} \cdot][\mathrm{M}]+k_{\mathrm{y}}[\mathrm{Y} \cdot][\mathrm{M}]
$$

where $k_{\mathrm{cx}}$ and $k_{\mathrm{cy}}$ are the rate constants for intramolecular monocyclization and bicyclization, respectively, and $k_{\mathrm{y}}$ is the rate constant of the intermolecular propagation of the monocyclized radical IV.

Assuming stationary state for both radical concentrations, $[\mathrm{X} \cdot]$ and $[\mathrm{Y} \cdot]$, their ratio is givne by,

$$
\frac{[\mathrm{Y} \cdot]}{[\mathrm{X} \cdot]}=\frac{k_{\mathrm{cx}}}{k_{\mathrm{cy}}+k_{\mathrm{y}}[\mathrm{M}]}
$$

consequently, the molar ratio of the unsaturated and saturated monomer units is expressed as,

$$
\frac{f_{\mathrm{u}}}{f_{\mathrm{s}}}=\frac{\mathrm{d} U / \mathrm{d} t}{\mathrm{~d} S / \mathrm{d} t}=\frac{k_{\mathrm{x}}+k_{\mathrm{y}}[\mathrm{Y} \cdot] /[\mathrm{X} \cdot]}{k_{\mathrm{cx}}+k_{\mathrm{cy}}[\mathrm{Y} \cdot] /[\mathrm{X} \cdot]}[\mathrm{M}]
$$

When eq 9 is substituted into eq 10 , we obtain,

$$
\frac{f_{\mathrm{u}}}{f_{\mathrm{s}}}=\frac{[\mathrm{M}]^{2}+\left(r_{\mathrm{c} 1}+r_{\mathrm{c} 2}\right)[\mathrm{M}]}{r_{\mathrm{c} 1}\left([\mathrm{M}]+2 r_{\mathrm{c} 2}\right)}
$$

where $r_{\mathrm{c} 1}$ and $r_{\mathrm{c} 2}$ are $k_{\mathrm{cx}} / k_{\mathrm{x}}$ and $k_{\mathrm{cy}} / k_{\mathrm{y}}$, respectively. Since $f_{\mathrm{u}}+f_{\mathrm{s}}=1, f_{\mathrm{u}}$ and $f_{\mathrm{s}}$ are expressed as functions of the monomer concentration, as in eq 12 and 13 .

$$
\begin{aligned}
& f_{\mathrm{u}}=\frac{[\mathrm{M}]^{2}+\left(r_{\mathrm{c} 1}+r_{\mathrm{c} 2}\right)[\mathrm{M}]}{[\mathrm{M}]^{2}+\left(2 r_{\mathrm{c} 1}+r_{\mathrm{c} 2}\right)[\mathrm{M}]+2 r_{\mathrm{c} 1} r_{\mathrm{c} 2}} \\
& f_{\mathrm{s}}=\frac{r_{\mathrm{c} 1}\left([\mathrm{M}]+2 r_{\mathrm{c} 2}\right)}{[\mathrm{M}]^{2}+\left(2 r_{\mathrm{c} 1}+r_{\mathrm{c} 2}\right)[\mathrm{M}]+2 r_{\mathrm{c} 1} r_{\mathrm{c} 2}}
\end{aligned}
$$

When $r_{\mathrm{c} 1}>r_{\mathrm{c} 2}$, eq 12 simplifies to eq 14 at a low monomer concentration. Thus,

$$
\frac{[\mathrm{M}]}{2 f_{\mathrm{u}}}=[\mathrm{M}]+r_{\mathrm{c} 2}
$$

Figure 3 shows the relationships between $[\mathrm{M}]_{0} /$ $2 f_{\mathrm{u}}$ and $[\mathrm{M}]_{0}$ for three polymers. The plot for VA satisfies eq 14, while the plots for VCA and VMA do not agree with eq 14. Thus, the assumption that $r_{\mathrm{c} 1}>r_{\mathrm{c} 2}$ is not valid for VCA and VMA. Therefore, an appropriate value of $r_{\mathrm{c} 2}\left(\fallingdotseq 0.10 \mathrm{~mol} \mathrm{dm}^{-3}\right)$ is substituted in eq 12 and $r_{\mathrm{c} 1}$ values is derived by the curve fitting method.

Figure 4 shows the theoretical curves of eq 12 in which the $r_{\mathrm{c} 1}$ values used are 20 for VA, 0.60 for VCA and $0.22 \mathrm{~mol} \mathrm{dm}^{-3}$ for VMA, and the $r_{\mathrm{c} 2}$ value is $0.10 \mathrm{~mol} \mathrm{dm}^{-3}$ for each of three polymers. Figure 4 also shows the theoretical curve of eq 1 for VMA, as a broken line, where $r_{\mathrm{c}}$ is $0.19 \mathrm{~mol} \mathrm{dm}^{-3}$. 


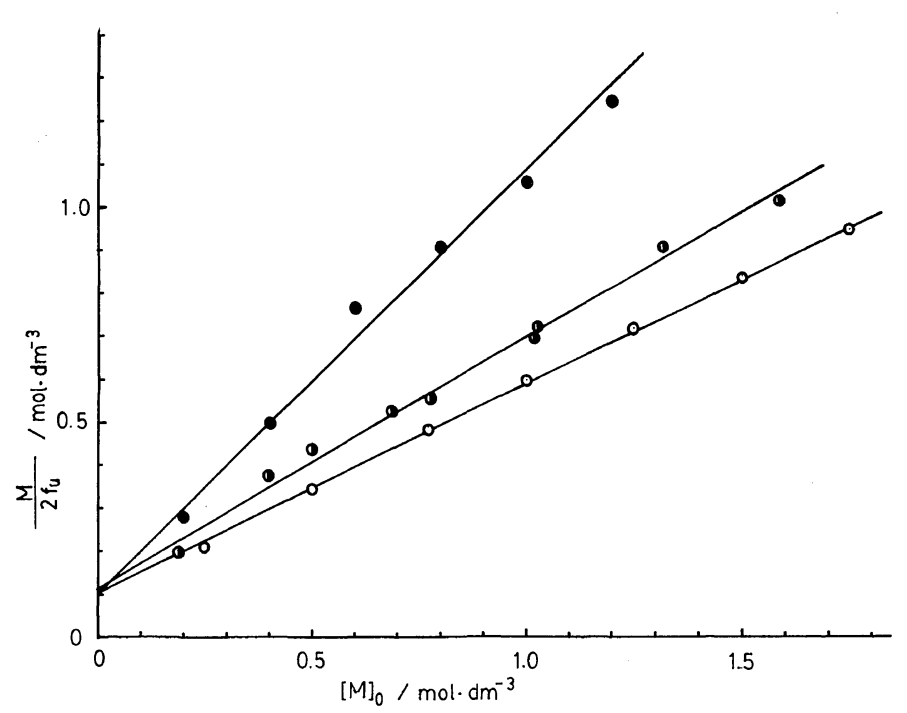

Figure 3. Relationships between $[\mathrm{M}]_{0} / 2 f_{\mathrm{u}}$ and $[\mathrm{M}]_{0}$ : polymerization conditions and symbols are the same as in Figure 2.

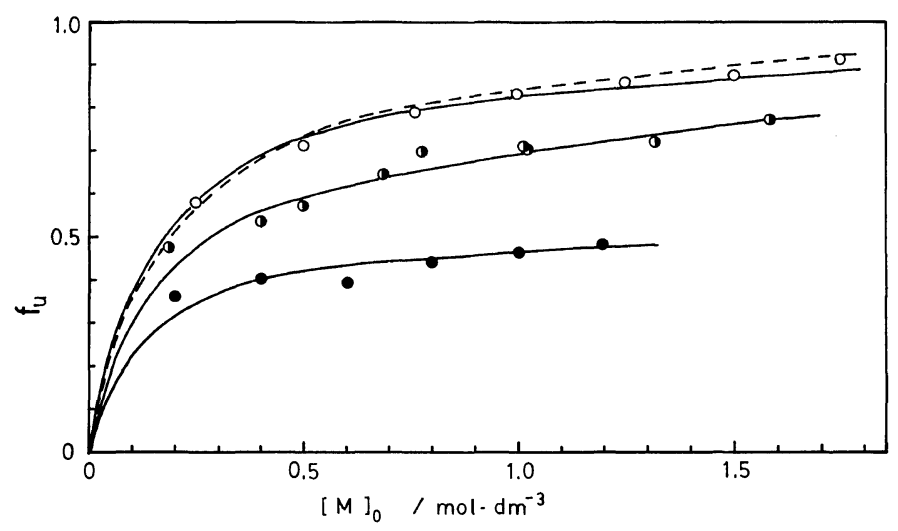

Figure 4. Effect of initial monomer concentration $[\mathrm{M}]_{0}$ on the fraction of residual double bond per monomer unit $f_{\mathrm{u}}$ : solid curves show the theoretical plots of eq 12 where $r_{\mathrm{c} 1}$ and $r_{\mathrm{c} 2}$ used are 20 and 0.10 for - VA, 0.60 and 0.10 for $\bigcirc \mathrm{VCA}$, and 0.22 and 0.10 for $\bigcirc \mathrm{VMA}$; broken line shows the theoretical plots of

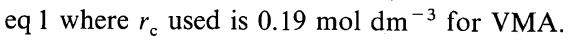

Both the theoretical curves for eq 1 and 12 for VMA agree with the experimental data in the same order. Thus, the cyclization mechanism of VMA polymerization cannot be determined on the basis of kinetics. However, the theoretical curves of eq 12 for VA and VCA are in good agreement with the experimental plots.

Thus, the proposed bicyclization mechanism is sufficient as an explanation of the polymerization of VA and VCA. In addition, the proposed mechanism may also apply to the polymerization of VMA since there is no proof of a $\gamma$-lactone structure in the polymer. Guaita et al. have proposed a monocyclization mechanism for the polymerization of VMA and calculated the ratio of the propagation rate constants of cyclic radicals toward methacrylic and vinyl groups to be $0.63 .{ }^{5}$ However, it doesn't seem reasonable that a vinyl group would be reactive toward a radical intermolecularly more than a methacrylic group on the basis of copolymerization data.

The extent of cyclization of VA is less than that of 
divinyl ether; their cyclization parameters are reported to be $r_{\mathrm{c} 1}=100$ and $r_{\mathrm{c} 2}=4.2 \mathrm{~mol} \mathrm{dm}^{-3}$, respectively. ${ }^{13}$ The difference in the $r_{\mathrm{c} 1}$ and $r_{\mathrm{c} 2}$ values of VA and divinyl ether are explained by the difference in the ease of internal rotation of the ester and ether units. A substitutent at the $\alpha$-position in acrylate lowers markedly the monocyclization ratio. It is suggested that steric factors are more important in intramolecular monocyclization than in intermolecular propagation. On the other hand, intramolecular bicyclization is little affected by the $\alpha$-substituent in acrylate as can be seen from eq 6 in Scheme I. The bicyclization ratios for VA, VCA, and VMA are estimated to be about the same, i.e., $r_{\mathrm{c} 2}=0.1$.

\section{Composition of Structural Units in the Polymers}

The saturated monomer units in the polymers are formed through monocyclization and bicyclization reactions. When mole fractions of saturated monomer units formed by monocyclization and bicyclization are designated as $f_{\mathrm{s} 1}$ and $f_{\mathrm{s} 2}$, respectively, the total fraction of saturated monomer units is $f_{\mathrm{s}}$ in eq 15 .

$$
f_{\mathrm{s}}=f_{\mathrm{s} 1}+f_{\mathrm{s} 2}
$$

The ratio $f_{\mathrm{s} 1} / f_{\mathrm{s} 2}$ is estimated by eq 16 .

$$
\frac{f_{\mathrm{s} 1}}{f_{\mathrm{s} 2}}=\frac{k_{\mathrm{cx}}[\mathrm{X} \cdot]}{k_{\mathrm{cy}}[\mathrm{Y} \cdot]}=1+\frac{[\mathrm{M}]}{r_{\mathrm{c} 2}}
$$

If $f_{\mathrm{s} 1}$ and $f_{\mathrm{s} 2}$ are introduced from eq 13,15 , and 16 , then,

$$
\begin{aligned}
& f_{\mathrm{s} 1}=\frac{r_{\mathrm{c} 1}}{[\mathrm{M}]+2 r_{\mathrm{c} 1}} \\
& f_{\mathrm{s} 2}=\frac{r_{\mathrm{c} 1} r_{\mathrm{c} 2}}{\left([\mathrm{M}]+2 r_{\mathrm{c} 1}\right)\left([\mathrm{M}]+r_{\mathrm{c} 2}\right)}
\end{aligned}
$$

When mole fractions of linear, monocyclic and bicyclic units are denoted by $f_{\mathrm{L}}, f_{\mathrm{C} 1}$, and $f_{\mathrm{C} 2}$, the sum of the fractions is unity,

$$
f_{\mathrm{L}}+f_{\mathrm{C} 1}+f_{\mathrm{C} 2}=1
$$

These fractions are shown in eq 20,21, and 22 as follows,

$$
\begin{aligned}
& f_{\mathrm{L}}=\frac{f_{\mathrm{u}}-\left(f_{\mathrm{s} 1}-f_{\mathrm{s} 2}\right)}{f_{\mathrm{u}}+f_{\mathrm{s} 2}} \\
& f_{\mathrm{C} 1}=\frac{f_{\mathrm{s} 1}-f_{\mathrm{s} 2}}{f_{\mathrm{u}}+f_{\mathrm{s} 2}}
\end{aligned}
$$

$$
f_{\mathrm{C} 2}=\frac{f_{\mathrm{s} 2}}{f_{\mathrm{u}}+f_{\mathrm{s} 2}}
$$

The molar ratio of each fraction is thus,

$$
f_{\mathrm{L}}: f_{\mathrm{C} 1}: f_{\mathrm{C} 2}=[\mathrm{M}]\left([\mathrm{M}]+r_{\mathrm{c} 2}\right): r_{\mathrm{c} 1}[\mathrm{M}]: r_{\mathrm{c} 1} r_{\mathrm{c} 2}
$$

Figure 5 shows the effect of monomer concentration on the mole fraction of each structural unit calculated from eq 23 . At a higher monomer concentration, the linear structure is predominant in poly(VMA), while the monocyclic unit is predominant in poly(VA). Poly(VCA) contains both linear and monocyclic units to about the same extent. A bicyclic structure can be observed only in the polymers obtained at a monomer concentration lower than $0.2 \mathrm{~mol} \mathrm{dm}^{-3}$.

\section{Copolymerization of $V C A$}

VCA was copolymerized with methyl acrylate (MA), vinyl acetate (VAc) and maleic anhydride (MAn) to determine if the cyclization ratios were influenced by the presence of monoolefins. Roovers and Smets have shown an equation of cyclocopolymerization in which the cyclization ratio changed not only with comonomer composition but also with absolute concentration. ${ }^{1}$ Table III summarizes

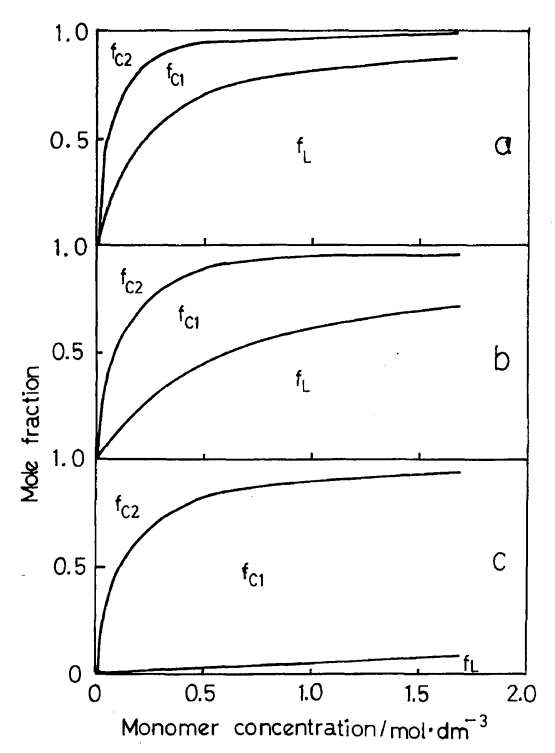

Figure 5. Dependence of monomer concentration on the structural units in the polymers obtained: $f_{\mathrm{L}}$, linear unit; $f_{\mathrm{c} 1}$, monocyclized unit; $f_{\mathrm{c} 2}$, bicyclized unit: a, poly(VMA); b, poly(VCA); c, poly(VA). 
W. FUKUDA et al.

Table III. Copolymerization of VCA with monoolefins ${ }^{\mathrm{a}}$

\begin{tabular}{|c|c|c|c|c|c|c|}
\hline$\left[\mathrm{M}_{1}\right]$ & {$\left[\mathrm{M}_{2}\right]$} & \multirow{2}{*}{$\frac{\text { Time }}{\min }$} & \multirow{2}{*}{$\frac{\text { Yield }}{w \mathrm{wt} \%}$} & \multirow{2}{*}{$\frac{\mathrm{Cl} \text { in copolymer }}{\mathrm{b}}$} & \multirow{2}{*}{$m_{1}$} & \multirow{2}{*}{$f_{\mathrm{v}}^{\mathrm{c}}$} \\
\hline \multicolumn{2}{|c|}{$\mathrm{mol} \mathrm{dm}^{-3}$} & & & & & \\
\hline VCA & MA & & & & & \\
\hline 0.41 & 0.23 & 20 & 6.4 & 25.80 & 0.942 & 0.57 \\
\hline 0.41 & 0.39 & 26 & 7.3 & 24.21 & 0.860 & 0.64 \\
\hline 0.41 & 0.81 & 25 & 4.7 & 21.52 & 0.726 & 0.74 \\
\hline 0.41 & 1.59 & 40 & 5.5 & 19.79 & 0.642 & 0.85 \\
\hline 0.16 & 1.35 & 30 & 5.2 & 17.22 & 0.539 & 0.92 \\
\hline 0.30 & 1.21 & 30 & 5.4 & 19.97 & 0.643 & 0.80 \\
\hline 0.51 & 1.00 & 35 & 8.5 & 21.59 & 0.731 & 0.76 \\
\hline 0.84 & 0.78 & 25 & 5.7 & 24.69 & 0.886 & 0.77 \\
\hline 1.02 & 0.50 & 20 & 6.6 & 25.78 & 0.942 & 0.74 \\
\hline 1.23 & 0.30 & 17 & 6.1 & 26.27 & 0.971 & 0.74 \\
\hline 0.80 & 1.63 & 30 & 5.4 & 21.69 & 0.735 & 0.95 \\
\hline 0.32 & 0.65 & 37 & 8.4 & 21.56 & 0.728 & 0.71 \\
\hline 0.13 & 0.26 & 37 & 6.4 & 21.28 & 0.716 & 0.58 \\
\hline VCA & VAc & & & & & \\
\hline 0.58 & 0.65 & 20 & 4.2 & 26.29 & 0.963 & 0.75 \\
\hline 0.50 & 0.20 & 20 & 6.3 & 26.83 & 1.000 & 0.64 \\
\hline 0.50 & 0.43 & 25 & 6.4 & 27.09 & 1.000 & 0.65 \\
\hline 0.50 & 1.10 & 15 & 2.9 & 25.52 & 0.926 & 0.75 \\
\hline 0.50 & 2.17 & 16 & 1.9 & 24.41 & 0.869 & 0.83 \\
\hline 0.05 & 0.95 & 50 & 1.9 & 20.76 & 0.693 & 0.66 \\
\hline 0.10 & 0.91 & 40 & 2.3 & 22.65 & 0.780 & 0.66 \\
\hline 0.15 & 0.83 & 30 & 3.3 & 23.23 & 0.807 & 0.69 \\
\hline 0.20 & 0.80 & 40 & 4.9 & 24.76 & 0.888 & 0.62 \\
\hline VCA & MAn & & & & & \\
\hline 0.40 & 0.40 & 30 & 6.6 & 25.59 & 0.940 & 0.54 \\
\hline 0.40 & 0.68 & 30 & 5.8 & 25.28 & 0.926 & 0.53 \\
\hline 0.40 & 1.19 & 35 & 5.6 & 23.71 & 0.850 & 0.52 \\
\hline 0.40 & 1.85 & 38 & 7.0 & 22.74 & 0.805 & 0.53 \\
\hline
\end{tabular}

a Copolymerization was conducted at $60^{\circ} \mathrm{C}$ in benzene solution with $1.0 \times 10^{-2} \mathrm{~mol} \mathrm{dm}^{-3} \mathrm{AIBN}$.

b Elemental analysis.

c Residual double bond per VCA monomer unit in a copolymer, as determined by the ICl titrimetric method.

the results of the copolymerizations.

Figure 6 shows copolymer composition as a function of monomer composition. Apparent monomer reactivity ratios determined by curve fitting method are $r_{1}=25, r_{2}=0.01$ for VCA-VAc, $r_{1}=15, r_{2}=0$ for VCA-MAn, and $r_{1}=7, r_{2}=0.1$ for VCA-MA, where $M_{1}$ is VCA and $M_{2}$ is a monoolefin. The high reactivity of the VCA monomer suggests that intermolecular propagation takes place between the growing polymer radical and the $\alpha$-chloroacrylic group of VCA rather than the vinyl group.

Figure 7 shows the effect of monoolefin addition on the reciprocal of the cyclization fraction of VCA in the copolymer. The addition of VAc or MA decreases the cyclization fraction $\left(1-f_{\mathrm{u}}\right)$, while the addition of MAn shows no effect on the cyclization fraction of VCA.

In the copolymerization of VCA with monoolefins, the reactions of Scheme II may proceed with the reactions of homopolymerization as shown in Scheme I. When cyclization reaction is influenced only by a monoolefin through the reaction of eq 24 , a decrease in the cyclization fraction will be proportional to $1 / r_{1}$ for each monomer, i.e., $\mathrm{MA}>$ MAn $>$ VAc. However, the result is MA $>$ VAc $\gg$ 


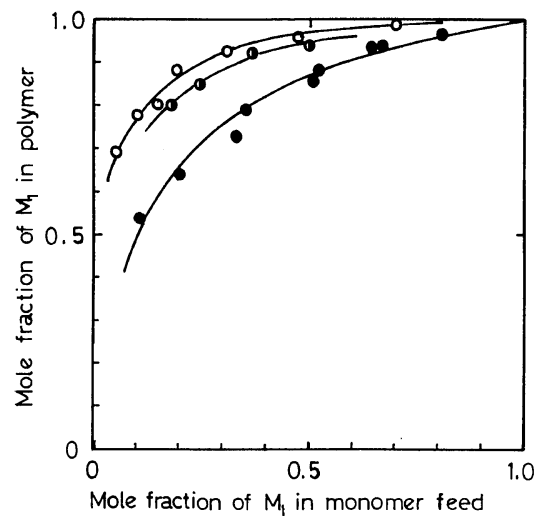

Figure 6. Copolymer composition curves of VCA with $\bigcirc \mathrm{VAc}, \mathrm{MAn}$, and MA: [AIBN], $1.0 \times 10^{-2} \mathrm{~mol}$ $\mathrm{dm}^{-3}$ in benzene at $60^{\circ} \mathrm{C} ; \mathrm{M}_{1}$, VCA.

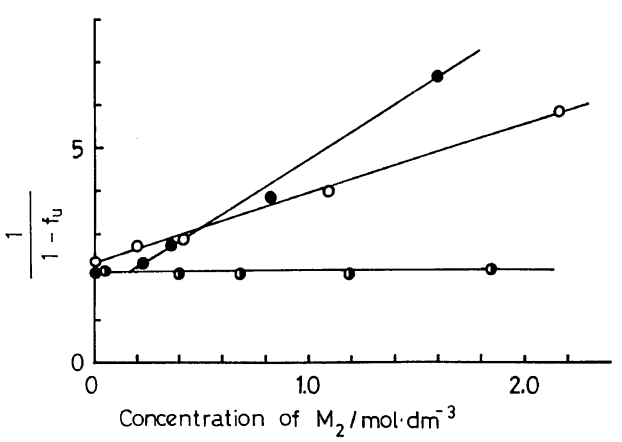

Figure 7. Effect of adding monoolefins on the reciprocal of cyclized fraction of VCA in the copolymers: symbols are the same as in Figure $6 ;\left[\mathrm{M}_{1}\right], 0.41 \mathrm{~mol} \mathrm{dm}^{-3}$ for $M_{2}=M A, 0.50 \mathrm{~mol} \mathrm{dm}^{-3}$ for $M_{2}=V A c, 0.40 \mathrm{~mol}$ $\mathrm{dm}^{-3}$ for $\mathrm{M}_{2}=$ MAn.

MAn. Consequently, the cyclization reaction of eq 27 is considered analogous to the cyclocopolymerization of divinyl ether with MAn. ${ }^{16}$ In this case, a decrease in the cyclization fraction is proportional to $k_{22} / k_{\mathrm{c} 2} \quad\left(=r_{2} k_{2 \mathrm{x}} / k_{\mathrm{c} 2}\right)$. The $k_{22} / k_{\mathrm{c} 2}$ value are estimated from the monomer reactivity ratios of monoolefins $\mathrm{M}_{2}$ in the copolymerization with VAc, i.e., 9 for MA,${ }^{17} 1$ for VAc and 0.003 for MAn. ${ }^{18}$. This order is comparable to the $r_{2}$ values in the copolymerization of VCA with $\mathrm{M}_{2}$, i.e., 0.1 for MA, 0.01 for VAc, and 0 for MAn. Both orders of the monomer reactivity ratios agree with the experimental results, $\mathrm{MA}>\mathrm{VAc} \gg \mathrm{MAn}$.

Accordingly, the presence of monoolefins in the cyclopolymerization of VCA affects the extent of cyclization with regard to both factors, $1 / r_{1}$ and $r_{2}$

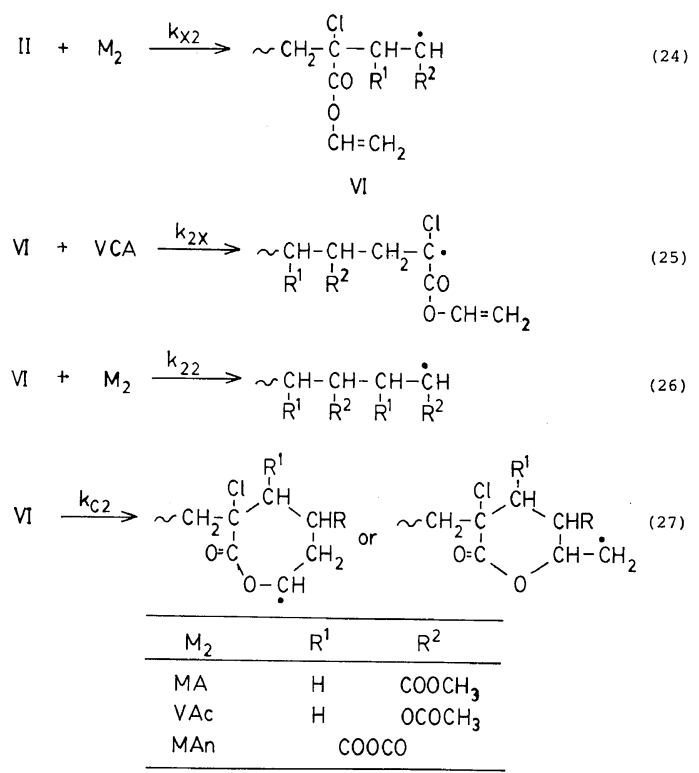

Scheme II.

where $r_{1}$ and $r_{2}$ are the copolymerization parameters of VCA and monoolefins. Although a kinetic equation for cyclocopolymerization cannot be solved, the results of copolymerization give additional support to the cyclization mechanism proposed.

In conclusion, vinyl esters of acrylic, methacrylic, and $\alpha$-chloroacrylic acid are suggested to polymerize with radical initiators during the process of monocyclization between a growing polymer radical and pendent vinyl group of a penultimate monomer unit and bicyclization between a monocyclized radical and vinyl group of a terminal unit.

\section{REFERENCES}

1. J. Roovers and G. Smets, Makromol. Chem., 60, 89 (1963).

2. I. A. Arbuzova and E. N. Rostovskii, J. Polym. Sci., 52, 325 (1961).

3. L. Trossarelli, M. Guaita, and A. Priola, Makromol. Chem., 100, 147 (1967).

4. W. Fukuda, M. Nakao, K. Okumura, and H. Kakiuchi, J. Polym. Sci., A-1, 10, 237 (1972).

5. M. Guaita, G. Camino, and L. Trossarelli, Makromol. Chem., 146, 133 (1971).

6. G. B. Butler, J. Polym. Sci., Polym. Symp., No. 64, 71 (1978).

7. S. R. Johns, R. I. Willing, S. Middleton, and A. K. Ong, J. Macromol. Sci., Chem., A10, 869 (1976). 
8. M. Tsukino and T. Kunitake, Macromolecules, 12, 387 (1979).

9. C. S. Marvel, J. Am. Chem. Soc., 62, 3495 (1940).

10. K. Chikanishi, T. Tsuruta, and J. Furukawa, Makromol. Chem., 84, 158 (1964).

11. L. J. Bellamy and R. L. Williams, J. Chem. Soc., 1957, 4294.

12. B. Wesslen and R. W. Lenz, Macromolecules, 4, 20 (1971).

13. M. Guaita, G. Camino, and L. Trossarelli, Makromol. Chem., 130, 243, 252 (1969); ibid., 131,
237 (1970)

14. C. Aso, S. Ushio, and M. Sogabe, Makromol. Chem., 100, 100 (1967).

15. M. Tsukino and T. Kunitake, Polym. J., 13, 657 (1981).

16. J. M. Barton, G. B. Butler, and R. C. Chapin, J. Polym. Sci., A, 3, 501 (1965).

17. L. P. Witnauer, N. Watkins, and W. S. Prot, $J$. Polym. Sci., 20, 213 (1956).

18. M. C. de Wilde and G. Smets, J. Polym. Sci., 5, 253 (1950). 